\title{
HOMOMORPHISMS OF JORDAN RINGS OF SELF-ADJOINT ELEMENTS
}

\author{
BY \\ N. JACOBSON AND C. E. RICKART
}

In a previous paper [4](1) we have defined a special Jordan ring to be a a subset of an associative ring which is a subgroup of the additive group and which is closed under the compositions $a \rightarrow a^{2}$ and $(a, b) \rightarrow a b a$. Such systems are also closed under the compositions $(a, b) \rightarrow a b+b a=\{a, b\}$ and $(a, b, c) \rightarrow a b c+c b a$. The simplest instances of special Jordan rings are the associative rings themselves. In our previous paper we studied the (Jordan) homomorphisms of these rings. These are the mappings $J$ of associative rings such that

$$
\begin{gathered}
(a+b)^{J}=a^{J}+b^{J}, \quad\left(a^{2}\right)^{J}=\left(a^{J}\right)^{2}, \\
(a b a)^{J}=a^{J} b^{J} a^{J} .
\end{gathered}
$$

A second important class of special Jordan rings is obtained as follows. Let $\mathfrak{A}$ be an associative ring with an involution $a \rightarrow a^{*}$, that is, a mapping $a \rightarrow a^{*}$ such that

$$
\begin{gathered}
(a+b)^{*}=a^{*}+b^{*}, \quad(a b)^{*}=b^{*} a^{*}, \\
a^{* *}=a .
\end{gathered}
$$

Let $\mathfrak{F}$ denote the set of self-adjoint elements $h=h^{*}$. Then $\mathfrak{F C}$ is a special Jordan ring. In this paper we shall study the homomorphisms of the rings of this type. It is noteworthy that the Jordan rings of this type include those of our former paper $\left({ }^{2}\right)$.

In our first paper we developed two methods for determining the Jordan homomorphisms of the rings $\mathfrak{A}$ : a matrix method and a Lie ring method. In this paper we obtain an analogue of the matrix method for the rings $\mathfrak{F}$. Our principal result (Theorem 4 ) is that if $\mathfrak{A}$ is a matrix ring $\mathfrak{S}_{n}, n \geqq 3$, with an involution such that $e_{i l}^{*}=e_{i i}, i=1, \cdots, n$, and every element of $\mathfrak{H C}$ is of the form $a+a^{*}$, then any Jordan homomorphism of $\mathcal{F}$ can be extended to an associative homomorphism of $\mathfrak{A}$. This result can be extended to locally matrix rings and in this form it is applicable to involutorial simple rings with minimal one-sided ideals. We also obtain the Jordan isomorphisms of the Jordan ring of self-adjoint elements of an involutorial primitive ring with minimal one-sided ideals onto a second Jordan ring of the same type.

Presented to the Society, September 7, 1951; received by the editors July 20, 1951.

(1) Numbers in brackets refer to the bibliography at the end of the paper.

(2) See the remark following Theorem 5 below. 
1. Canonical involutions in matrix rings. We recall that a ring $\mathfrak{A}$ with an identity 1 is a matrix ring $\mathfrak{S}_{n}$ if $\mathfrak{A}$ contains a set of matrix units $\left\{e_{i j}\right\}, i, j$ $=1,2, \cdots, n$, such that

$$
e_{i j} e_{k l}=\delta_{j k} e_{i l}, \quad \sum_{1}^{n} e_{i i}=1 .
$$

The ring $\mathfrak{S}$ is the subring of $\mathfrak{A}$ of elements commuting with all the $e_{i j}$. As is well known, every element of $\mathfrak{A}$ can be written in one and only one way as $\sum \xi_{i j} e_{i j}, \xi_{i j} \in \mathfrak{S}$.

We assume now that $\mathfrak{A}=\mathfrak{S}_{n}$ has an involution $x \rightarrow x^{*}$ such that the $e_{i i}$ are self-adjoint elements. We wish to determine the form of the involution in terms of the $e_{i j}$ and the coefficient ring $\mathfrak{S}$.

Lemma 1. If $\mathfrak{A}=\mathfrak{S}_{n}$ and $x \rightarrow x^{*}$ is an involution such that $e_{i \mathfrak{i n}}^{*}=e_{i i}$, $i=1,2, \cdots, n$, then there exists an involution $\alpha \rightarrow \bar{\alpha}$ in $\subseteq$ and self-adjoint elements $\gamma_{i} \in \subseteq\left(\bar{\gamma}_{i}=\gamma_{i}\right)$ having inverses such that

$$
x^{*}=\sum \gamma_{j}^{-1} \bar{\xi}_{i j} \gamma_{i} e_{j i}, \quad x=\sum \xi_{i j} e_{i j} .
$$

Conversely, a mapping $x \rightarrow x^{*}$ of the form (4) is an involution such that $e_{\mathfrak{i t}}^{*}=e_{i i}$.

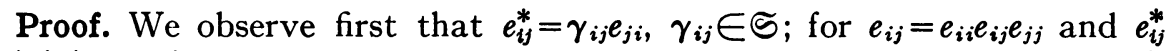
$=e_{j j}^{*} e_{i j}^{*} e_{i 1}^{*}=e_{j j} e_{i j}^{*} e_{i i}$. Let $\gamma_{11}=\gamma_{i}, \gamma_{1 i}=\gamma_{i}^{\prime}, i=1,2, \cdots, n$. Since $e_{11}=e_{1 i} e_{i 1}, e_{11}^{*}$ $=e_{i 1}^{*} e_{11}^{*}=\gamma_{i} \gamma_{i}^{\prime} e_{11}$. It follows that $e_{j j}=\gamma_{i} \gamma_{i}^{\prime} e_{j j}$ and summation on $j$ gives $\gamma_{i} \gamma_{i}^{\prime}$ $=1$. Similarly we can start from $e_{i i}=e_{i 1} e_{1 i}$ and prove $\gamma_{i}^{\prime} \gamma_{i}=1$. Hence $\gamma_{i}^{\prime}$ $=\gamma_{i}^{-1}$. Also it is clear that $\gamma_{1}=1$. If $\xi \in S, \xi e_{11}=e_{11}\left(\xi e_{11}\right) e_{11}$ and this shows that $\left(\xi e_{11}\right)^{*}=\bar{\xi} e_{11}$. The element $\bar{\xi}$ is uniquely determined; hence $\xi \rightarrow \bar{\xi}$ is an involution in $\subseteq$. Now $\xi_{i j} e_{i j}=e_{i 1}\left(\xi_{i j} e_{11}\right) e_{1 j}$. Hence

$$
\begin{aligned}
\left(\xi_{i j} e_{i j}\right)^{*} & =e_{1 j}^{*}\left(\xi_{i j} e_{11}\right)^{*} e_{i 1}^{*}=e_{1 j}^{*}\left(\bar{\xi}_{i j} e_{11}\right) e_{i 1}^{*} \\
& =\gamma_{j}^{-1} e_{j 1}\left(\bar{\xi}_{i j} e_{11}\right) \gamma_{i} e_{1 i} \\
& =\gamma_{j}^{-1} \bar{\xi}_{i j} \gamma_{i} e_{j i}
\end{aligned}
$$

and (4) holds. Since $e_{i 1}=e_{i 1}^{* *}=\left(\gamma_{i} e_{1 i}\right)^{*}=\gamma_{i}^{-1} \bar{\gamma}_{i} \gamma_{1} e_{i 1}=\gamma_{i}^{-1} \bar{\gamma}_{i} e_{i 1}, \bar{\gamma}_{i}=\gamma_{i}$. Conversely we can verify that if $\alpha \rightarrow \bar{\alpha}$ is an involution in $\subseteq$ and the $\gamma_{i}$ are selfadjoint elements of $\subseteq_{\text {, then }}(4)$ defines an involution in $\Im_{n}$ such that $e_{i 1}^{*}=e_{i i}$.

An involution of the form (4) in a matrix ring will be called canonical. Of course, this is no restriction if $n=1$. In the sequel we shall require $n \geqq 3$. We consider now some special cases.

A. Let $\mathfrak{A}$ be an involutorial simple ring with minimum condition for right ideals. Then $\mathfrak{A}=\Delta_{n}$ where $\Delta$ is a division ring. We observe that the involution is canonical. This follows easily from known results except when $n=2 m$, $\Delta=\Phi$ is a field, and the involution is symplectic, that is, has the form $x \rightarrow q^{-1} x^{\prime} q$ 
where $x^{\prime}$ denotes the transpose of $x$ and

$$
\left.q=\left(\begin{array}{lll}
\sigma & & \\
& \sigma & \\
& \ddots & \\
& &
\end{array}\right)\right\} m, \quad \sigma=\left(\begin{array}{rr}
0 & 1 \\
-1 & 0
\end{array}\right) .
$$

In this case we can regard $\mathfrak{A}$ as $\Im_{m}$ where $\subseteq=\Phi_{2}$ and if we introduce the involution $\alpha \rightarrow \bar{\alpha}=\sigma^{-1} \alpha^{\prime} \sigma$ in $\Phi_{2}$, then the given involution in $\mathfrak{A}$ is canonical with all the $\gamma_{i}=1$.

B. Let $\mathfrak{X}$ be a vector space over a division ring $\Delta$ which possesses an involution $\alpha \rightarrow \bar{\alpha}$. We assume, moreover, that there is defined a scalar product $f=(x, y)$ relative to $\alpha \rightarrow \bar{\alpha}$ in $\mathfrak{X}$. This means that $(x, y)$ is a function from $\mathfrak{X} \times \mathfrak{X}$ to $\Delta$ such that

$$
\begin{aligned}
\left(x, y_{1}+y_{2}\right) & =\left(x, y_{1}\right)+\left(x, y_{2}\right), \\
\left(x_{1}+x_{2}, y\right) & =\left(x_{1}, y\right)+\left(x_{2}, y\right), \\
(\alpha x, y) & =\alpha(x, y), \\
(x, \alpha y) & =(x, y) \bar{\alpha} .
\end{aligned}
$$

Suppose that $(x, y)$ is hermitian in the sense that $(y, x)=(\overline{x, y})$ and nondegenerate in the sense that $(x, z)=0$ for all $x$ only if $z=0$. Let $\&_{f}$ be the ring of all linear transformations $t$ in $\mathfrak{X}$ which have adjoints relative to $f$; that is, if $t \in \mathfrak{R}_{f}$, then there exists a $t^{*}$ such that $(x t, y)=\left(x, y t^{*}\right)$ holds for all $x, y$ in $\mathfrak{X}$. Then $t \rightarrow t^{*}$ is an involution in $\ell_{f}$. We shall study such involutions later. At this point we note that if $\mathfrak{X}$ has an orthonormal basis (for finite combinations) $\left\{e_{\mu}\right\},\left(e_{\mu}, e_{\nu}\right)=\delta_{\mu \nu}$, and $\operatorname{dim} \mathfrak{X}>1$, then $\ell_{f}$ is a nontrivial matrix ring and $t \rightarrow t^{*}$ is canonical. If $\operatorname{dim} \mathfrak{X}<\infty$, this has been proved in A. Hence assume $\operatorname{dim} \mathfrak{X}=\infty$. In this case, for every integer $n$ we can decompose the basis $\left\{e_{\mu}\right\}$ into $n$ disjoint sets $\left\{e_{\mu}^{(k)}\right\}, k=1,2, \cdots, n$, where $\mu$ ranges over the same set of indices in each case. Let $e_{i j}$ be the linear transformation defined by

$$
e_{\mu}^{(k)} e_{i j}=\delta_{k i} e_{\mu}^{(j)},
$$

$i, j=1,2, \cdots, n$. Then the $e_{i j}$ form a system of matrix units for $\ell_{f}$ and $e_{i j}^{*}=e_{j i}$. In particular $e_{\mathfrak{u}}^{*}=e_{i i}$ so that $t \rightarrow t^{*}$ is canonical.

A similar discussion applies to the case in which $\Delta=\Phi$ a field, $\bar{\alpha}=\alpha$, and $f$ is an alternate scalar product in the sense that $(x, x)=0$ for all $x$, provided that $\mathfrak{X}$ has a symplectic basis. By this we mean a basis $\left\{d_{\mu}, e_{\mu}\right\}$ in $\mathfrak{X}$ such that

$$
\begin{array}{ll}
\left(d_{\mu}, d_{\nu}\right)=0=\left(e_{\mu}, e_{\nu}\right), \\
\left(d_{\mu}, e_{\nu}\right)=0, \\
\left(d_{\mu}, e_{\mu}\right)=1=-\left(e_{\mu}, d_{\mu}\right) .
\end{array} \quad \mu \neq \nu,
$$


If $\ell_{f}$ denotes the ring of linear transformations having adjoints and $\operatorname{dim} \mathfrak{X}>2$, then $\mathfrak{R}_{f}=\mathfrak{S}_{n}, n>1$, and $t \rightarrow t^{*}$, the adjoint of $t$, is a canonical involution.

C. Let $\mathfrak{B}$ be the ring of all bounded operators in a Hilbert space $\mathfrak{S}$ (not necessarily separable). Here we have the fundamental scalar product $(x, y)$ and the involution $t \rightarrow t^{*}$ in $\mathfrak{B}$. While there exists no orthonormal basis in the algebraic sense, there do exist complete orthonormal systems $\left\{e_{\mu}\right\}$ for $\mathfrak{W}$. If we take into account the completeness of $\mathfrak{E}$, we can use a complete orthonormal system in the same way as the orthonormal basis was used above to prove that $t \rightarrow t^{*}$ is canonical.

D. Let $\mathfrak{A}$ be a ring of linear transformations in a Hilbert space $\mathfrak{W}$ which is a factor in the sense of Murray and von Neumann [6]. Again $t \rightarrow t^{*}$ is an involution in $\mathfrak{A}$. Factors of type I are already covered in the preceding example. On the other hand, if $\mathfrak{A}$ is a factor of type II or III, then it is not difficult to obtain a decomposition of Hilbert space of the form $\mathfrak{S}=\mathfrak{M}_{1}+\mathfrak{M}_{2}$ $+\cdots+\mathfrak{M}_{n}$ where $n$ is any integer and the $\mathfrak{M}_{i}$, which "belong to $\mathfrak{A}^{\prime}$ in the sense of Murray and von Neumann [6], are pairwise equivalent. Then for each $j=1, \cdots, n$ there exists a partially isometric operator $e_{1 j}$ in $\mathfrak{A}$ with $\mathfrak{M}_{1}$ as its initial set and $\mathfrak{M}_{j}$ as its final set. For $i, j=1, \cdots, n$, define $e_{i j}$ $=e_{1 i}^{*} e_{1 j}$. Obviously $\left\{e_{i j}\right\}$ is a system of matrix units and $e_{\mathfrak{i j}}^{*}=e_{\mathfrak{H}}$. Hence $\mathfrak{A}$ is of the form $\mathfrak{S}_{n}$ and $t \rightarrow t^{*}$ is canonical.

2. The Jordan ring of self-adjoint elements of a matrix ring with a canonical involution. Let $\mathfrak{A}$ be a matrix ring $\mathfrak{S}_{n}$ with a canonical involution $x \rightarrow x^{*}$ and denote by $\mathfrak{H C}$ the Jordan ring of self-adjoint elements relative to this involution. Then $h=\sum \alpha_{i j} e_{i j} \in \mathfrak{F}$ if and only if

$$
\alpha_{i j}=\gamma_{i}^{-1} \bar{\alpha}_{j i} \gamma_{j}
$$

holds for all $i, j$. In particular, if $\alpha$ is any element of $\mathfrak{S}$, then

$$
\alpha[i j]=\alpha e_{i j}+\gamma_{i}^{-1} \bar{\alpha} \gamma_{i} e_{j i}=\alpha e_{i j}+\left(\alpha e_{i j}\right)^{*}
$$

is in $\mathcal{H}$. With this notation the element $h$ can be written as

$$
h=\sum_{i} \alpha_{i i} e_{i i}+\sum_{i<j} \alpha_{i j}[i j]
$$

where $\gamma_{t}^{-1} \bar{\alpha}_{t \imath} \gamma_{t}=\alpha_{t \mathfrak{t}}$. We note also that

$$
\alpha[i j]=\gamma_{j}^{-1} \bar{\alpha} \gamma_{i}[j i] .
$$

We shall not attempt to list all the Jordan relations connecting the elements $\alpha_{!}[i j]$ but note only two of these which occur most frequently:

$$
\begin{aligned}
\{\alpha[i j], \beta[j k]\} & =\alpha \beta[i k], & i, j, k \neq . \\
\{\alpha[i j], \beta[j i]\} & =\alpha \beta[i i]+\beta \alpha[j j], & i \neq j .
\end{aligned}
$$

We prove first the following result. 
Lемма 2. If $n \geqq 2$, then the enveloping associative ring of $\operatorname{HC}$ is $\mathfrak{S}_{n}$ itself.

Proof. Let $\alpha$ be any element of $\subseteq$ and let $i \neq j$. Since $e_{i i}$ and $\alpha[i j] \in \mathcal{H}$, $\alpha e_{i j}=e_{i i} \alpha[i j]$ is in the enveloping ring. Hence so is $\alpha e_{i i}=\left(\alpha e_{i j}\right) e_{j i}$. This implies the lemma.

If $\mathfrak{F}$ is the set of self-adjoint elements relative to an involution in a ring $\mathfrak{A}$, then we shall say that $\mathfrak{H C}$ is trace-valued $[5, \mathrm{p} .8]$ if every $h \in \mathcal{H}$ has the form $a+a^{*}, a \in \mathfrak{A}$. This will always be the case if $\mathfrak{A}$ admits the operator $1 / 2$, that is, if $2 x=a$ has a unique solution $(1 / 2) a$ for every $a \in \mathfrak{A}$. There are instances in which $\mathfrak{H C}$ is trace-valued even when $\mathfrak{A}$ is of characteristic two. From the form (9) of $h \in \mathcal{H C}$ of $\mathfrak{S}_{n}$, it is clear that the set of self-adjoint elements of a canonical involution of a matrix ring is trace-valued if and only if this holds for the sets of self-adjoint elements of $\subseteq$ relative to the involutions $\xi \rightarrow \gamma_{i}^{-1} \bar{\xi} \gamma_{i}$. Since $\eta$ is self-adjoint relative to $\xi \rightarrow \gamma_{i}^{-1} \xi \gamma_{i}$ if and only if $\gamma_{i} \eta$ is self-adjoint relative to $\xi \rightarrow \bar{\xi}$, it follows that a necessary and sufficient condition that $\mathfrak{F}$ be trace-valued is that the set of self-adjoint elements of $\subseteq$ relative to $\xi \rightarrow \bar{\xi}$ is trace-valued.

We recall that a subset $\mathfrak{F}$ of a special Jordan ring $\mathfrak{H C}$ is a (Jordan) ideal provided (1) $\Im$ is a subgroup of the additive group, (2) $\Im$ contains $\{a, z\}=a z$ $+z a$ for all $a \in \mathfrak{H}, z \in \Im,(3) \mathfrak{F}$ contains $z^{2}, a z a, z a z$ for $a \in \mathfrak{F}, z \in \mathfrak{F}$. If $\mathfrak{H}$ admits $1 / 2$, then (3) is superfluous.

TheOREM 1. Let $\mathfrak{A}=\Im_{n}$ have a canonical involution and let $\mathfrak{H C}$ be the Jordan ring of self-adjoint elements. Assume that $n \geqq 3$ and that for every self-adjoint ideal $\mathfrak{B}$ of $\mathfrak{A}\left(\mathfrak{B}^{*}=\mathfrak{B}\right), \mathfrak{B} \cap \mathfrak{H C}$ is trace-valued. Then a subset $\mathfrak{Y}$ of $\mathfrak{H C}$ is a Jordan ideal in $\mathfrak{H C}$ if and only if $\mathfrak{S}=\mathfrak{B} \cap \mathcal{H C}$ where $\mathfrak{B}$ is a self-adjoint ideal of the associative ring $\mathfrak{A}$.

Proof. Obviously if $\mathfrak{B}$ is an ideal in $\mathfrak{A}$, then $\mathfrak{B} \cap \mathfrak{H}$ is a Jordan ideal in $\mathfrak{H}$. Therefore let $\Im$ be an arbitrary Jordan ideal in $\mathcal{H}$. We can assume $\Im \neq(0)$. Let $\Lambda$ denote the subset of $\subseteq$ of elements which appear as coefficients of the elements of $\Im$. We prove first that $\Lambda$ is an ideal in the associative ring $\mathfrak{S}$ and hence that $\mathfrak{B}=\Lambda_{n}$ is an ideal in $\mathfrak{A}$.

Let $h=\sum \eta_{i j} e_{i j}$ be an arbitrary element of $\Im$. Since $\eta_{i i} e_{i i}=e_{i i} h e_{i i}$, we have $\eta_{i i} e_{i i} \in \mathcal{S}$. Furthermore, if $i \neq j$, then $\eta_{i i}[i j]=\left\{\eta_{i i} e_{i i}, u_{i j}\right\}$ where $u_{i j}=1[i j]$. Hence $\eta_{i i}[i j] \in \Im$. Also if $i \neq j, \eta_{i j}[i j]=e_{i i} h e_{j j}+e_{j j} h e_{i i} \in \Im$. It follows that $\Lambda$

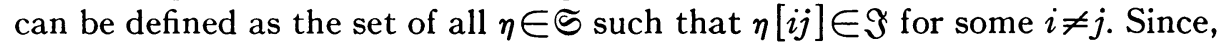
by (11), $\eta[k j]=\left\{u_{k j}, \eta[i j]\right\}, i, j, k \neq$, and $\eta[i k]=\left\{\eta[i j], u_{j k}\right\}$ it follows that if $\eta \in \Lambda$ then $\eta[k l] \in \Im$ for arbitrary $k, l, k \neq l$. An immediate corollary of this result is that $\Lambda$ is a group under addition. Furthermore, if $\eta \in \Lambda$ and $\alpha \in \mathbb{S}$, then for $i, j, k \neq,(\eta \alpha)[i k]=\{\eta[i j], \alpha[j k]\}$ is in $\Im$. Hence $\eta \alpha \in \Lambda$ and similarly $\alpha \eta \in \Lambda$ so that $\Lambda$ is an ideal in $\subseteq$. If we take into account (10), we see that $\Lambda$ is self-adjoint relative to the involution $\xi \rightarrow \bar{\xi}$ of $\mathfrak{S}$. It follows that $\mathfrak{B}=\Lambda_{n}$ is a self-adjoint ideal of $\mathfrak{A}$. 
It is obvious that $\mathfrak{Y} \subset \mathfrak{B} \cap \mathfrak{F}$. Hence let $h=\sum \lambda_{i i} e_{i i}+\sum_{i<j} \lambda_{i j}[i j] \in \mathfrak{B} \cap \mathfrak{F}$. We have seen that the $\lambda_{i j}[i j] \in \mathcal{S}$. Hence it remains to show that $\lambda e_{i i} \in \mathcal{S}$ if $\lambda \in \Lambda$ and $\bar{\lambda}=\gamma_{i} \lambda \gamma_{i}^{-1}$. Now since $\mathfrak{B} \cap \mathcal{H C}$ is trace-valued, we can write $\lambda=\eta$ $+\gamma_{i}^{-1} \bar{\eta} \gamma_{i}, \eta \in \Lambda$. Hence $\lambda e_{i i}=\eta[i i]$. Since $\eta[i j] \in \Im$, it follows from (12) that

$$
\eta[i i]=e_{i i}\left\{\eta[i j], u_{j i}\right\} e_{i i}
$$

is in $\Im$. This completes the proof.

CoROLlaRy. Let $\mathfrak{A}=\mathfrak{S}_{n}$ be a simple ring with a canonical involution. Assume that $n \geqq 3$ and that $\mathcal{H C}$ is trace-valued. Then $\mathcal{H C}$ is a simple Jordan ring.

Besides the matrix rings we shall be concerned in the sequel with generalizations of these rings defined as follows:

Definition. A ring $\mathfrak{A}$ with an involution will be called locally canonicalmatrix if every finite subset of its elements can be imbedded in a self-adjoint matrix subring in which the induced involution is canonical. The ring $\mathfrak{A}$ will be called locally of degree at least $n$ if the matrix subrings can always be chosen of degree $n$ or greater.

The preceding results can be extended to locally canonical-matrix rings. We note explicitly the following two theorems.

Theorem 2. Let $\mathfrak{A}$ be a locally canonical-matrix ring, locally of degree at least two. Then $\mathfrak{A}$ is the enveloping associative ring of its Jordan ring $\mathfrak{H C}$ of self-adjoint elements.

This follows directly from the lemma.

THEOREM 3. Let $\mathfrak{A}$ be a simple ring which is locally canonical-matrix Assume that $\mathfrak{A}$ is locally of degree at least three and that $\mathfrak{H C}$ is trace-valued. Then $\mathfrak{H C}$ is a simple Jordan ring.

Proof. Let $\mathfrak{M}$ be one of the matrix rings referred to in the definition and let $e$ be the identity of $\mathfrak{M}$ and $\left\{e_{i j}\right\}$ a set of matrix units such that $e_{i \mathfrak{i n}}^{*}=e_{i t}$. Now we can replace $\mathfrak{M}$ by the larger ring $e \mathfrak{A} e$. This is a self-adjoint matrix subring with $\left\{e_{i j}\right\}$ as matrix units. Since $e_{i t}^{*}=e_{i i}$, the induced involution is canonical in $e \mathfrak{A} e$. It is well known that $e \mathfrak{A} e$ is simple. If $k \in e \mathfrak{A} e \cap \mathfrak{H}$, then $k=a+a^{*}=e a e+e a^{*} e$. Hence $e \mathfrak{A} e \cap \mathfrak{H C}$ is trace-valued. The result now follows from the corollary.

3. Jordan homomorphisms of $\mathfrak{H C}$ of a matrix ring. In this section we prove the analogue of the matrix theorem (Theorem 7) of our former paper.

THEOREM 4. Let $\mathfrak{A}=\mathfrak{S}_{n}$ be a matrix ring with a canonical involution. Assume that $n \geqq 3$ and that the Jordan ring $\mathfrak{F C}$ is trace-valued. Then any Jordan homomorphism of $\mathfrak{H C}$ can be extended in one and only one way to an associative homomorphism of $\mathfrak{A}$.

Proof. Let $h \rightarrow h^{J}$ be a homomorphism of $\mathfrak{H C}$ and let $\varepsilon$ denote the envelop- 
ing associative ring of the image $\mathfrak{T C}^{J}$. We recall that $J$ maps orthogonal idempotents into orthogonal idempotents. More generally, if $e, a \in \mathfrak{K}, e$ idempotent, and $e a=0=a e$ holds in $\mathfrak{A}$, then $\left(^{3}\right) e^{J} a^{J}=0=a^{J} e^{J}$. If $\alpha$ is any element of S, $e_{i i} \alpha[i i] e_{i i}=\alpha[i i]$. Hence $e_{\mathfrak{H}}^{J} \alpha[i i]^{J} e_{\mathfrak{H}}^{J}=\alpha[i i]^{J}$ and

$$
e_{i i}^{J} \alpha[i i]^{J}=\alpha[i i]^{J}=\alpha[i i]^{J} e_{i i}^{J} .
$$

Next let $i \neq j$. Then $\alpha[i j]=\left\{\alpha[i j], e_{j j}\right\}$ so that $\alpha[i j]^{J}=\left\{\alpha[i j]^{J}, e_{j j}^{J}\right\}$. Multiplication of this equation on the left by $e_{\mathfrak{u}}^{J}$ gives

$$
\stackrel{J}{e_{i i} \alpha}[i j]^{J}=\stackrel{J}{e_{i i} \alpha}[i j]^{J} e_{j j}^{J} .
$$

Similarly $e_{t 3}^{J} \alpha[i j]^{J} e_{j j}^{J}=\alpha[i j]^{J} e_{j j}^{J}$. Hence

$$
\stackrel{J}{e_{i i} \alpha}[i j]^{J}=\stackrel{J}{e_{i i} \alpha[i j]^{J}} e_{i j}^{J}=\alpha[i j]^{J} e_{i j}^{J}
$$

holds for all $i, j$.

As before, set $u_{i j}=1$ [ij]. If $i, j, k$ are all different, then $u_{i k}=u_{i j} u_{j k} e_{k k}$ $+e_{k k} u_{j k} u_{i j}$. Therefore

$$
\stackrel{J}{u_{i k}}=\stackrel{J}{u_{i j}} \underset{j k}{J} e_{k k}^{J}+\stackrel{J}{e_{k k}} \underset{u_{j k}}{J} u_{i j}^{J}
$$

and

$$
\underset{e_{i i} u_{i k}^{J}}{J}=e_{i i}^{J} u_{i j}^{J} u_{j k}^{J} e_{k k}^{J} .
$$

If we define $g_{i j}=e_{\mathfrak{k}}^{J} u_{\mathfrak{y}}^{J}$ for $i \neq j$, then (13), (14), and the orthogonality of the $e_{\mathrm{t}}^{J}$ imply that

$$
g_{i j} g_{k l}=\delta_{j k} g_{i l}
$$

holds for $i \neq j, k \neq l$, and $i \neq l$.

We prove next that $g_{i j} g_{j i}=e_{\mathfrak{i}}^{J}$. We note first that $u_{i j} u_{j i}=e_{i i}+e_{j j}=u_{j i} u_{i j}$. This implies that the element $z=u_{\mathfrak{i}}^{J} u_{j \mathfrak{j}}^{J}-u_{f f}^{J} u_{i j}^{J}$ is in the center( $\left.{ }^{(}\right)$of $\varepsilon$ and $z^{2}=0$. Now set $\left(e_{i i}+e_{j j}\right)^{J}-u_{i j}^{J} u_{\mathcal{H}}^{J}=\left(u_{i j} u_{j i}\right)^{J}-u_{i j}^{J} u_{j h}^{J}=y$. Then $\left(u_{i j} u_{j i}\right)^{J}-u_{j l}^{J} u_{i j}^{J}$ $=y+z$. Hence $\left({ }^{5}\right)$

$$
y(y+z)=\left[\left(u_{i j} u_{j i}\right)^{J}-u_{i j}^{J} u_{j i}^{J}\right]\left[\left(u_{i j} u_{j i}\right)^{J}-u_{j i}^{J} u_{i j}^{J}\right]=0 .
$$

Therefore $y^{2}=-y z$ and

$$
y^{3}=-y^{2} z=y z^{2}=0
$$

Now observe that

$$
y e_{i i}^{J}=\left(e_{i i}^{J}+\stackrel{J}{e_{j i}}-\stackrel{J}{u_{i j}} u_{j i}^{J}\right) e_{i i}^{J}=\stackrel{J}{e_{i i}}-\stackrel{u_{i j}}{J} u_{j i}^{J} e_{i i}^{J}=e_{i i}^{J}-g_{i j} g_{j i}
$$

(3) The proof in $[4$, p. 482] applies here without essential modification.

(4) The proof in $[4$, pp. 481, 482] applies here without essential modification.

(5) The proof in $[4, \mathrm{p} .482]$ applies here without essential modification. 
and similarly $e_{i t}^{J} y=e_{\mathfrak{i}}^{J}-g_{i j} g_{j i}$. On the other hand,

$$
\begin{aligned}
\left(g_{i j} g_{j i}\right)^{2} & =g_{i j} g_{j i} g_{i j} g_{j i}=e_{i i}^{J} u_{i j}^{J} u_{j i}^{J} u_{i j}^{J} u_{j i}^{J} \\
& =e_{i i}^{J}\left(u_{i j} u_{j i} u_{i j}\right)^{J} u_{j i}^{J}=e_{i i}^{J} u_{i j} u_{j i}^{J}=g_{i j} g_{j i}
\end{aligned}
$$

and this implies that $e_{i j}^{J}-g_{i j} g_{j i}$ is idempotent. Thus $y e_{i t}^{J}=y^{2} e_{i 4}^{J}=y^{3} e_{i 4}^{J}=0$ by (16). Hence $g_{i j} g_{j i}=e_{i t}^{J}$. Then if we set $g_{i i}=e_{k i}^{J}$, (15) will hold for all

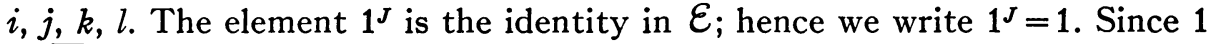
$=\sum e_{i i}$

$$
\sum g_{i i}=1 .
$$

Therefore $\left\{g_{i j}\right\}$ is a set of matrix units for $\varepsilon$.

We can represent $\varepsilon$ as $\mathfrak{F}_{n}$ where $\mathfrak{F}$ is the subring of $\mathcal{E}$ of the elements which commute with the $g_{i j}$. Now let $\alpha$ be any element of $\mathfrak{S}$ and write

$$
\alpha[i j]^{J}=\sum_{k, l} \alpha[i j]_{k l} g_{k l}, \quad \alpha[i j]_{k l} \in \mathfrak{F} .
$$

Then by (13)

$$
\alpha[i j]^{J} g_{j j}=\alpha[i j]_{i j} g_{i j}
$$

holds for all $i, j$. We next define $\alpha^{\tau_{i j}}=\alpha[i j]_{i j}$ and proceed to show that $\alpha \rightarrow \alpha^{\tau_{i j}}$ is a homomorphism of $\subseteq$ into $\mathfrak{F}$ which is independent of $i, j$ if $i \neq j$. If we apply $J$ to (11), we obtain

$$
\alpha[i j]^{J} \beta[j k]^{J}+\beta[j k]^{J} \alpha[i j]^{J}=(\alpha \beta)[i k]^{J}
$$

for $i, j, k$ all different. Since $\alpha[i j] e_{k k}=0=e_{k k} \alpha[i j]$, right multiplication of the last equation by $g_{k k}$ gives

$$
\alpha[i j]^{J} \beta[j k]^{J} g_{k k}=(\alpha \beta)[i k]^{J} g_{k k} .
$$

This implies

$$
\alpha^{\tau_{i j} \beta^{\tau_{j i}}}=(\alpha \beta)^{\tau_{i k}}
$$

if $i, j, k$ are unequal. Now recall that $1[i j]=u_{i j}$ so that $1[i j]^{J} g_{j j}=u_{i j}^{J} g_{j j}=g_{i j}$. Comparison with (18) gives $1[i j]_{i j}=1$; hence $1^{\tau^{i j}}=1$. If we put $\beta=1$ in (19), we now obtain $\alpha^{\tau_{i j}}=\alpha^{\tau_{i k}}$. Similarly $\alpha^{\tau_{i j}}=\alpha^{\tau_{k i}}$ and hence

$$
\alpha^{\tau_{i j}}=\alpha^{\tau_{k l}}, \quad i \neq j, k \neq l .
$$

It follows that $\alpha^{\tau}=\alpha^{\tau_{i j}}$ is independent of $i, j$ if $i \neq j$ and, by (19), $\alpha \rightarrow \alpha^{\tau}$ is a homomorphism of $\mathfrak{S}$ into $\mathfrak{F}$.

Now for $i \neq j,\left\{\alpha[i j], u_{j i}\right\}=\alpha[i i]+\alpha[j j]$. Hence $\left\{\alpha[i j]^{J}, u_{j\}}^{J}\right\}=\alpha[i i]^{J}$ $+\alpha[j j]]^{J}$. Since $\alpha[j j] e_{i i}=0=e_{i i} \alpha[j j]$, this gives

$$
\alpha[i j]^{J} u_{j i g_{i i}}^{J}+u_{j i}^{J}[i j]^{J} g_{i i}=\alpha[i i]^{J} g_{i i} .
$$


Since $\alpha[i j]^{J} u_{j i}^{J} g_{i i}=\alpha[i j]^{J} g_{j i}=\alpha[i j]^{J} g_{j j} g_{j i}$, we obtain from (18) that $\alpha[i j]^{J} u_{j l}^{J} g_{i i}$ $=\alpha^{\tau} g_{i i}$. Hence

$$
\alpha^{\tau} g_{i i}+\stackrel{J}{u_{j i} \alpha[i j]^{J} g_{i i}}=\alpha[i i]^{J} g_{i i} .
$$

Since $\alpha[i j]=\left(\gamma_{j}^{-1} \bar{\alpha} \gamma_{i}\right)[j i]$ and $u_{j i}=\left(\gamma_{i}^{-1} \gamma_{j}\right)[i j], \alpha[i j]^{J} g_{i i}=\left(\gamma_{j}^{-1} \bar{\alpha} \gamma_{i}\right)^{\tau} g_{j i}$ and $u_{j i}^{J} g_{j i}=\left(\gamma_{i}^{-1} \gamma_{j}\right)^{\tau} g_{i i}$. Hence (21) becomes

$$
\alpha^{\tau} g_{i i}+\left(\gamma_{j}^{-1} \bar{\alpha} \gamma_{i}\right)^{\tau} g_{i i}=\alpha[i i]^{J} g_{i i} .
$$

For arbitrary $x=\sum \xi_{i j} e_{i j}$ in $\mathfrak{A}$ we define $x^{T}=\sum \xi_{i j}^{\tau} g_{i j}$. Evidently $x \rightarrow x^{T}$ is a homomorphism of $\mathfrak{A}$ into $\mathcal{E}$. We wish to show that $T$ coincides with $J$ on FC. Let $i \neq j$ and consider $\alpha[i j]^{J}$. Since $\alpha[i j]^{J} g_{k k}=0$ for $k \neq i, j$,

$$
\begin{aligned}
\alpha[i j]^{J} & =\alpha[i j]^{J} 1=\alpha[i j]^{J} g_{j j}+\alpha[i j]^{J} g_{i i} \\
& =\alpha^{\tau} g_{i j}+\left(\gamma_{j} \bar{\alpha} \gamma_{i}^{-1}\right)^{\tau} g_{j i} \\
& =\alpha[i j]^{T} .
\end{aligned}
$$

Similarly $\alpha[i i]^{J}=\alpha[i i]^{J} g_{i i}=\alpha[i i]^{T}$ by (22). These two results show that $x^{J}=x^{T}$ if $x=a+a^{*}$. Since $\mathfrak{H C}$ is trace-valued this proves our assertion. Thus $T$ is an associative extension of $J$ to $\mathfrak{A}$. That the extension is unique is a consequence of the fact that $\mathfrak{A}$ is the enveloping associative ring of $\mathfrak{H C}$.

If we make use of the fact, established in the proof of Theorem 3, that if $\mathfrak{A}$ is locally canonical-matrix and $\mathfrak{H C}$ is trace-valued then we can suppose that $\mathfrak{H} \cap \mathfrak{M}$ is trace-valued for the required matrix subrings $\mathfrak{M}$ of $\mathfrak{A}$, we obtain the following extension of the matrix theorem.

Theorem 5. Let $\mathfrak{A}$ be a locally canonical-matrix ring. Assume that $\mathfrak{A}$ is locally of degree at least three and that $\mathfrak{H C}_{\mathrm{C}}$ is trace-valued. Then any Jordan homomorphism of $\mathfrak{H C}$ can be extended in one and only one way to an associative homomorphism of $\mathfrak{A}$.

Theorem 4 actually implies Theorem 7 of our previous paper [4] when $n \geqq 3$. This can be seen as follows. Let $\mathfrak{A}$ be an $n \times n$ matrix ring with $n \geqq 3$ and form $\mathfrak{B}=\mathfrak{A} \oplus \mathfrak{A}^{\prime}$ with involution $\left(a+b^{\prime}\right)^{*}=b+a^{\prime}$, where $\mathfrak{A}^{\prime}$ is anti-isomorphic to $\mathfrak{A}$ under the mapping $a \rightarrow a^{\prime}$. Let $\left\{e_{i j}\right\}$ be a system of matrix units in $\mathfrak{A}$ and define $f_{i j}=e_{i j}+e_{j l}^{\prime}$. Then $\left\{f_{i j}\right\}$ is an $n \times n$ system of matrix units in $\mathfrak{B}$ such that $f_{i j}^{*}=f_{j i}$. Hence the involution in $\mathfrak{B}$ is canonical. The Jordan ring $\mathfrak{H C}$ of self-adjoint elements in $\mathfrak{A}$ consists of all elements of the form $a+a^{\prime}$ and is Jordan isomorphic to $\mathfrak{A}$. Note that $\mathfrak{F C}$ is trace-valued since $a+a^{\prime}$ $=(a+0)+(a+0)^{*}$. Now, if $a \rightarrow a^{J}$ is a Jordan homomorphism of $\mathfrak{A}$, then $a+a^{\prime} \rightarrow a^{J}$ is a Jordan homomorphism of $\mathcal{H C}$ which, by Theorem 4 , can be extended to an associative homomorphism $T$ of $\mathfrak{B}$. The desired result now is immediate from Theorem 4 of $[4]\left({ }^{6}\right)$.

(6) Note that Theorems 5 and 6 of [4] apply trivially here to give the hypotheses of Theorem 4 of [4]. 
Theorem 7 of [4] is valid for $n \geqq 2$ while Theorem 4 above is not true for $n=2$. This is shown by an example in $[1$, pp. 156,157$]$.

4. Involutorial primitive rings with minimal ideals. In this section we shall consider the problem of determining the Jordan homomorphisms of the sets of self-adjoint elements of involutorial primitive rings with minimal onesided ideals. We first describe a method for obtaining the rings of this type. Let $\mathfrak{X}$ be a vector space over a division ring $\Delta$ which has an involution $\alpha \rightarrow \bar{\alpha}$ and let $f=(x, y)$ be nondegenerate hermitian or alternate scalar product in $\mathfrak{X}$. Let $\mathfrak{R}_{f}$ denote the ring of linear transformations $t$ in $\mathfrak{X}$ which possess adjoints $t^{*}:(x t, y)=\left(x, y t^{*}\right)$, and let $\mathfrak{F}_{f}$ be the subring of $\mathfrak{R}_{f}$ of transformations of finite rank ( $\mathfrak{X} t$ finite-dimensional). Then $t \rightarrow t^{*}$ is an involution in $\mathbb{R}_{f}$ and in $\mathfrak{F}_{f}$. It can be shown that any self-adjoint subring $\mathfrak{A}$ of $\mathfrak{R}_{f}$ containing $\mathfrak{F}_{f}$ is an involutorial primitive ring with minimal one-sided ideal and conversely every ring having this structure can be obtained in this way $\left({ }^{7}\right)$. There is therefore no loss in generality in dealing with the rings of linear transformations $\mathfrak{A}$ such that $\mathfrak{l}_{f} \supseteq \mathfrak{A} \supseteq \mathfrak{F}_{f}$ and $\mathfrak{A} *=\mathfrak{A}$. Let $\mathfrak{H C}$ be the Jordan ring of selfadjoint elements of $\mathfrak{A}$ and let $\mathfrak{H C}_{0}=\mathfrak{H C} \cap \mathfrak{F}_{f}$.

We consider first the case of involutorial simple rings with minimal onesided ideals. This amounts to assuming that $\mathfrak{A}=\mathfrak{F}_{f}, \mathfrak{F C}=\mathfrak{H}_{0}$. The basic result for the Jordan theory of $\mathfrak{F}_{f}$ is that $\mathfrak{F}_{f}$ is locally canonical-matrix. We proceed to the proof of this result. Since everything has been proved for finite-dimensional $\mathfrak{X}$ in our previous discussion, we assume from now on that $\operatorname{dim} \mathfrak{X}=\infty$.

If $\mathfrak{M}$ is a subspace of $\mathfrak{X}$, we define the orthogonal complement $\mathfrak{M}^{\perp}=\{x \mid(x, y)$ $=0, y \in \mathfrak{M}\}$. The subspace $\mathfrak{M}$ is nonisotropic, isotropic, or totally isotropic according as $\mathfrak{M} \cap \mathfrak{M}^{\perp}=(0), \mathfrak{M} \cap \mathfrak{M}^{\perp} \neq(0)$, or $\mathfrak{M} \subseteq \mathfrak{M}^{\perp}$. In general $\mathfrak{X} \neq \mathfrak{M} \oplus \mathfrak{M}^{\perp}$ even when $\mathfrak{M}$ is nonisotropic. However, if $\mathfrak{M}$ is a finite-dimensional nonisotropic subspace, then $\mathfrak{X}=\mathfrak{M} \oplus \mathfrak{M}^{\perp}[7$, Lemma 1.1]. The next lemma, which is fundamental for our purposes, is due to Kaplansky [5, Lemma 4]. We include the proof since the journal in which it appeared is not readily accessible.

LEMMA 3. Let $\mathfrak{M}$ be an arbitrary finite-dimensional subspace of $\mathfrak{X}$. Then there exists a finite-dimensional nonisotropic subspace of $\mathfrak{X}$ which contains $\mathfrak{M}$.

Proof. Choose a nonzero vector $x$ in $\mathfrak{M} \cap \mathfrak{M}^{\perp}$ and let $y$ be any vector such that $(x, y) \neq 0$. Let $\mathfrak{N}$ be the space spanned by $\mathfrak{M}$ and $y$. Then $\mathfrak{R} \cap \mathfrak{N}^{\perp}$ $\subset \mathfrak{M} \cap \mathfrak{M}^{\perp}$. A finite number of such steps completes proof of the lemma.

Theorem 6. Any involutorial simple ring with minimal one-sided ideals is locally canonical-matrix.

Proof. We take the ring to be $\mathfrak{F}_{f}$. Let $g_{1}, \cdots, g_{k}$ be a finite set of elements in $\mathfrak{F}_{f}$ and, by the preceding lemma, choose a finite-dimensional nonisotropic

(7) This follows from the isomorphism theorem for primitive rings with minimal ideals [2, p. 318]. A detailed proof will be given in [3, Chapter IX]. 
subspace $\mathfrak{M}$ in $\mathfrak{X}$ which contains the range of the linear transformations $g_{1}, \cdots, g_{k}$ and of the adjoints $g_{1}^{*}, \cdots, g_{\mathbf{k}}^{*}$. Denote by $\Re$ the set of $g \in \mathfrak{F}_{f}$ of the form $x g=\sum\left(x, a_{i}\right) b_{i}$ where $a_{i}$ and $b_{i}$ are in $\mathfrak{M}$. Since $x g^{*}= \pm \sum\left(x, b_{i}\right) a_{i}$, $g^{*} \in \Omega$ so that $\Omega$ is self-adjoint. Observe also that $\Omega$ contains the given $g_{i}$. Now let $u_{1}, \cdots, u_{n}$ be a basis for $\mathfrak{M}$. Since $\mathfrak{M}$ is nonisotropic, the scalar product $(x, y)$ is nondegenerate in $\mathfrak{M}$; therefore there exist $v_{1}, \cdots, v_{n} \in \mathfrak{M}$ such that $\left(u_{i}, v_{j}\right)=\delta_{i j}, i, j=1,2, \cdots, n$. Define $e_{i j}$ by $x e_{i j}=\left(x, v_{i}\right) u_{j}$. Then $\left\{e_{i j}\right\}$ is a set of matrix units for $\Omega$ and, in fact, $\Omega=\Delta_{n}$ [4, p. 490]. It follows from $A$ of $\$ 1$ that the involution is canonical in $\Omega$.

We remark that since $\mathfrak{X}$ is infinite-dimensional, we can take $n$ to be any finite number. The condition $\operatorname{dim} \mathfrak{X}$ infinite is equivalent to the assumption that $\mathfrak{A}$ does not satisfy the minimum condition for right (left) ideals. Our results therefore yield the following theorem.

TheOREM 7. Let $\mathfrak{A}$ be an involutorial simple ring possessing minimal onesided ideals but not satisfying the minimum condition for these ideals. Assume that the Jordan ring 30 of self-adjoint elements is trace-valued. Then $3 C$ is a simple Jordan ring and any Jordan homomorphism of $\mathfrak{H C}$ can be extended in one and only one way to an associative homomorphism of $\mathfrak{A}$.

We consider next an arbitrary primitive ring $\mathfrak{A}$ with minimal one-sided ideals. Take $\mathfrak{A}$ in the form $\mathfrak{R}_{f} \supseteq \mathfrak{A} \supseteq \mathfrak{F}_{f}, \mathfrak{A}^{*}=\mathfrak{A}$, and set $\mathfrak{F C}_{0}=\mathfrak{H C}_{\mathcal{C}} \cap \mathfrak{F}_{f}$, where $\mathfrak{H}$ is the Jordan ring of self-adjoint elements in $\mathfrak{A}$. We then have the following result.

Lemma 4. If $\mathfrak{H}_{0}$ is trace-valued, then it is a minimal Jordan ideal in $\mathfrak{H C}$ which is contained in every nonzero Jordan ideal of $\mathcal{H}$.

Proof. Let $\Im$ be a nonzero Jordan ideal in $\mathfrak{H}$. Since $\mathfrak{H}_{0}$ is a simple Jordan ring, we have only to prove that $\Im \cap \mathfrak{H}_{0} \neq(0)$. Let $b$ denote any nonzero element of $\Im$ and choose a vector $u$ such that $u b=v \neq 0$. We prove that there exists $h \in \mathcal{F}_{0}$ such that $u h=u$. First, if $(u, u) \neq 0$, define $h$ by $x h$ $=(x, u)(u, u)^{-1} u$. Then clearly $h \in \mathfrak{K}_{0}$ and $u h=u$. If $(u, u)=0$, choose a vector $w$ such that $(u, w)=1$ and define $t$ by $x t=(x, w) u$. In this case take $h=t+t^{*}$. Then again $h \in \mathcal{H}_{0}$ and $u h=u$. Now if $v h \neq 0$, then $h b h \in \mathcal{S} \cap \mathcal{C}_{0}$ and $u h b h$ $=v h \neq 0$ so that $h b h \neq 0$. Finally, if $v h=0$, then $\{b h\} \in \Im \cap \mathcal{F}_{0}$ and $u\{b h\}$ $=v \neq 0$ so that $\{b h\} \neq 0$.

The enveloping associative ring of the Jordan ring $\mathfrak{H}$ need not be equal to $\mathfrak{A}$. An example of this type can be obtained as follows. Let $\mathfrak{X}$ be a vector space with a denumerable basis over a field $\Phi$ and let $\mathfrak{l}_{f}$ be the ring of linear transformations in $\mathfrak{X}$ whose matrices are row and column finite relative to a certain basis for $\mathfrak{X}$, that is, there are only a finite number of nonzero coefficients in each row and column. The correspondence $(\alpha) \rightarrow(\alpha)^{\prime}$, the transpose of $(\alpha)$, defines an involution in $\ell_{f}$, and $\ell_{f}$ is a primitive ring with minimal one-sided ideals. The ideal $\mathfrak{F}_{f}$ corresponds to the set of matrices that have 
only a finite number of nonzero coefficients. Let $(\omega)$ be a skew-symmetric matrix with $n$ rows and columns such that $(\omega)^{2}=0$. Such matrices exist if $n \geqq 4$ and $\Phi$ is an algebraically closed field of characteristic 0 . Then the matrix

$$
\operatorname{diag}\{(\omega),(\omega),(\omega), \cdots\}
$$

with $(\omega)$ down the diagonal belongs to $\ell_{f}$. The ring $\mathfrak{A}$ generated by the corresponding linear transformation $w$ and $\mathfrak{F}_{f}$ is the set of mappings $\alpha w+g$, $\alpha \in \Phi, g \in \mathfrak{F}_{f} . \mathfrak{A}$ is self-adjoint and the set of self-adjoint elements of $\mathfrak{A}$ is the set $\mathfrak{F C}_{0}$ of self-adjoint elements of $\mathfrak{F}_{f}$. Hence the enveloping associative ring of $\mathfrak{H C}_{0}$ is $\mathfrak{F}_{f}$ which is properly contained in $\mathfrak{A}$.

The above example shows that, in order to obtain a relation between Jordan homomorphisms of $\mathfrak{H C}$ and associative homomorphisms of $\mathfrak{A}$, we need in general the additional condition that $\mathfrak{A}$ be equal to the enveloping associative ring $\varepsilon$ of $\mathfrak{F}$. On the other hand, since $\varepsilon \supseteq \mathscr{F}_{f}, \mathcal{E}$ is a primitive ring with minimal one-sided ideals which is clearly self-adjoint. Hence the condition $\Re=\mathcal{E}$ is a natural one.

THEOREM 8. Let $\mathfrak{A}$ and $\mathfrak{B}$ be involutorial primitive rings possessing minimal one-sided ideals and assume that $\mathfrak{A}$ and $\mathfrak{B}$ are not simple. Let $\mathfrak{H C}$ and $K$ be the Jordan rings of self-adjoint elements of $\mathfrak{A}$ and $\mathfrak{B}$ respectively. Assume that $\mathfrak{H C}$ and $K$ are trace-valued and that the enveloping associative rings are $\mathfrak{A}$ and $\mathfrak{B}$. Then any Jordan isomorphism of $\mathfrak{x C}$ onto $\Re$ can be extended in one and only one way to an associative isomorphism of $\mathfrak{A}$ onto $\mathfrak{B}$.

Proof. Take $\mathfrak{A}$ in the form $\mathfrak{R}_{f} \supseteq \mathfrak{A} \supseteq \mathfrak{F}_{f}, \mathfrak{A}^{*}=\mathfrak{A}$, and set $\mathfrak{F C}_{0}=\mathfrak{H C}_{\mathcal{C}} \cap \mathfrak{F}_{f}$, the set of self-adjoint elements in $\mathfrak{F}_{f}$. Similarly take $\mathfrak{B}$ in the form $\mathfrak{R}_{g} \supseteq \mathfrak{B} \supseteq \mathfrak{F}_{\theta}$, $\mathfrak{B}^{*}=\mathfrak{B}$, and set $K_{0}=K \cap \mathfrak{F}_{0}$. Let $h \rightarrow h^{J}$ be the Jordan isomorphism of $\mathfrak{F C}$ onto $K$. By Lemma $4, J$ also is a Jordan isomorphism of $\mathfrak{K}_{0}$ onto $K_{0}$. An application of Theorem 7 gives a unique extension of $J$ from $\mathfrak{F}_{0}$ to an associative isomorphism of $\mathfrak{F}_{f}$ onto $\mathfrak{F}_{g}$. Now there exists a unique extension of the isomorphism between $\mathfrak{F}_{f}$ and $\mathfrak{F}_{g}$ to an associative isomorphism $T$ between $\mathfrak{R}_{f}$ and $\mathfrak{R}_{0}\left[2\right.$, p. 318]. Define $J^{\prime}=J T^{-1}$. Then $J^{\prime}$ is a Jordan isomorphism of $\mathfrak{F}$ into $\mathfrak{R}_{f}$ which leaves elements of $\mathcal{H}_{0}$ fixed. Next we show that $J^{\prime}$ leaves every element of $\mathfrak{F C}$ fixed. Let $h \in \mathcal{H}$ and $h_{0} \in \mathfrak{H}_{0}$. Then $\left\{h h_{0}\right\} \in \mathfrak{F}_{0}$ so that $\left\{h h_{0}\right\}$ $=\left\{h h_{0}\right\}^{J^{\prime}}=\left\{h^{J^{\prime}} h_{0}\right\}$. Hence

$$
h h_{0}+h_{0} h=h^{J^{\prime}} h_{0}+h_{0} h^{J^{\prime}} .
$$

Since $h_{0} h h_{0} \in \mathfrak{F}_{0}$, we also have

$$
h_{0} h h_{0}=h_{0} h^{J^{\prime}} h_{0} .
$$

Now multiply (23) on the left by $h_{0}$ to obtain

$$
h_{0} h h_{0}+h_{0}^{2} h=h_{0} h^{J^{\prime}} h_{0}+h_{0}^{2} h^{J^{\prime}}=h_{0} h h_{0}+h_{0}^{2} h^{J^{\prime}} .
$$


Therefore

$$
h_{0}^{2}\left(h-h^{J^{\prime}}\right)=0 .
$$

Next let $u$ be any nonzero vector and, as in the proof of Lemma 4, choose $h_{0} \in \mathfrak{C}_{0}$ such that $u h_{0}=u$. Substitution in (24) gives $u\left(h-h^{J^{\prime}}\right)=0$. In other words, $h^{J^{\prime}}=h$. It follows that $h^{J}=h^{T}$ for all $h \in \mathfrak{F}$. Since $\mathfrak{A}$ and $\mathfrak{B}$ are the enveloping associative rings of $\mathfrak{H C}$ and $K$ respectively, it is immediate that $T$ is a unique extension of $J$ to an associative isomorphism of $\mathfrak{A}$ onto $\mathfrak{B}$.

The above proof actually gives us a slightly stronger result which we state as follows.

CoROllaRY. Let $\mathcal{H C}$ and $K$ be arbitrary Jordan rings of self-adjoint elements in $\mathfrak{R}_{f}$ and $\mathfrak{R}_{0}$ each of which contains all self-adjoint elements of finite rank. Then any Jordan isomorphism between $\mathfrak{H C}$ and $K$ has a unique extension to an associative isomorphism between $\mathfrak{R}_{f}$ and $\mathfrak{R}_{g}$.

\section{BIBLIOGRAPHY}

1. F. D. Jacobson and N. Jacobson, Classification and representation of semi-simple Jordan algebras, Trans. Amer. Math. Soc. vol. 65 (1949) pp. 141-169.

2. N. Jacobson, The radical and semi-simplicity for arbitrary rings, Amer. J. Math. vol. 67 (1945) pp. 300-320.

3. - Lectures in abstract algebra, vol. II, forthcoming.

4. N. Jacobson and C. E. Rickart, Jordan homomorphisms of rings, Trans. Amer. Math. Soc. vol. 69 (1950) pp. 479-502.

5. I. Kaplansky, Forms in infinite dimensional spaces, Anais da Academia Brasileira de Ciencias vol. 22 (1950) pp. 1-17.

6. F. J. Murray and J. von Neumann, On rings of operators, Ann. of Math. vol. 37 (1936) pp. 116-229.

7. C. E. Rickart, Isomorphic groups of linear transformations, Amer. J. Math. vol. 73 (1951) pp. 697-716.

Yale University,

New Haven, Conn. 